\title{
Image Processing Techniques: A Review
}

\section{Neetu Rani}

Computer Science Department, Chitkara University, Himachal Pradesh.

E-mail: neetu.rani@chitkarauniversity.edu.in

Published Online: June 28, 2017

The Author(s) 2017. This article is published with open access at www.chitkara.edu.in/publications

\begin{abstract}
In today's scenario image processing is one of the vast growing fields. It is a method which is commonly used to improve raw images which are received from various resources. It is a kind of signal processing. This paper provides an overview of image processing methods. The main concern of this paper is to define various techniques used in different phases of image processing.
\end{abstract}

Keywords: Image processing, Segmentation, Threshold, Acquisition, Enhancement.

\section{INTRODUCTION}

Image processing is spreading in various fields. Image processing is a method which is commonly used to improve raw images which are received from various resources [1]. It is a technique to transform an image into digital form and implement certain actions on it, in order to create an improved image or to abstract valuable information from it. It is a kind of signal dispensation where image is an input and output is also an image or features related with image. The purpose of image processing is distributed into several groups which are given below.

Visualization: Image processing is used to identify those objects which are not detectable.

Image sharpening and restoration: In image processing, various techniques are applied on the picture to produce a better image.

Image retrieval: By image processing user can detect only that portion of the picture which is relevant to the user.

Pattern measurement: Numerous elements in an image are measured.

Image Recognition: Substances in an image are recognized.

Image processing use mathematical procedures for processing of images. Two methods used for processing of images are analog image processing and digital image processing.
Journal on Today's Ideas Tomorrow's Technologies, Vol. 5, No. 1, June 2017 pp. $40-49$

CHITKARA 旬 


\section{Analog Image Processing}

This processing method use electrical signals for any change required in the picture. Analog processing includes two dimensional analog signals. In this approach images are modified by changing the electrical signal. It is mainly used for hard copies like as for printing purpose and for photography.

\section{Digital image processing}

In this technique processing of images are done by digital computers. Firstly via scanner-digitizer images are converted into digital form and then further processing is done on the images. Digital image processing uses many techniques like as correction, formatting of the data, enhance procedure to create picture with better quality [2]. Basically, there are mainly four operations used in digital image processing like as image preprocessing, segmentation of image, feature extraction, classification of images.

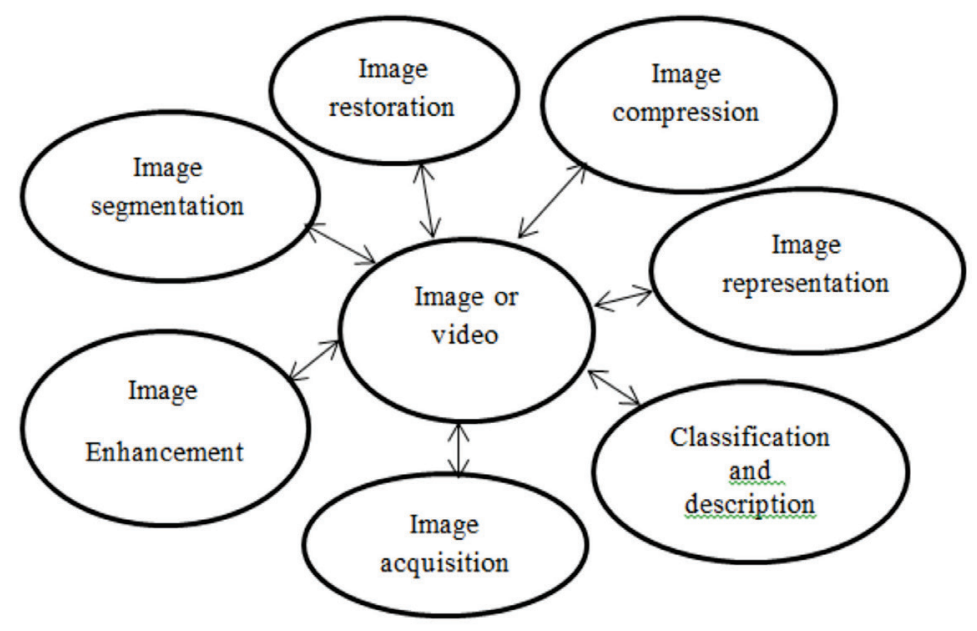

Figure 1: Techniques of Image Processing

\section{IMAGE SEGMENTATION}

Segmentation means partitioning of image into various regions or parts. In image segmentation, an image is divided into subparts according to the requirement of the user or the problem being solved [3]. It divides the image in to pixels. Image segmentation divides the image in such a way so that it becomes very accurate. Basically this approach is used for analysis of substances, borders and additional records which are relevant for processing [4]. The outcome of image segmentation is a set of sections that together cover the total image or group of contours removed from the image. The objective of segmentation is 
Rani, N.

to simplify or to modify the demonstration of picture in such a manner that is more significant and easy to evaluate. It produces the better appearance of image. Segmentation of images is done for compression of image, recognition of objects and for editing purpose. For image segmentation image thresholding methods are applied. Segmentation allocates label to each pixel in the image, such that pixel having similar label can share definite features [5].

\section{VARIOUS METHOD FOR IMAGE SEGMENTATION}

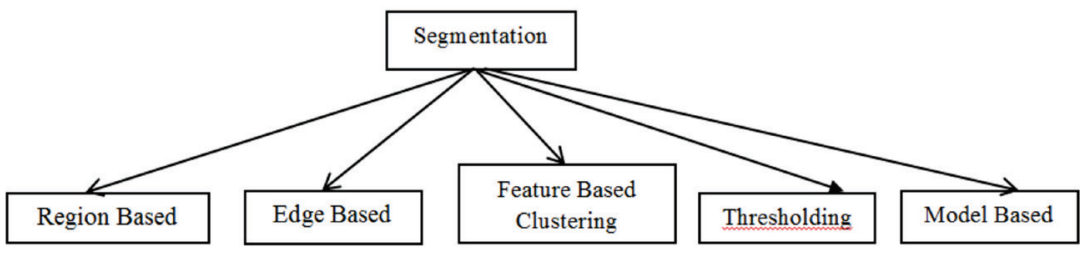

Figure 2: Image Segmentation Techniques

\section{Region Based}

This technique groups together certain objects used for segmentation [6] [7]. Region based segmentation technique used with this method. That region must be together with each other on which segmentation have to performed. It is also known as similarity based segmentation. The borders are recognized to perform segmentation. Every step takes at least one pixel for processing purpose. After applying the process color and texture of the image is altered and then a vector is created from the edge flow. Then further processing is applied on these edges [8].

\section{Edge based}

Another technique for segmentation is edge detection method. To identify dissimilarities from the image edges are identifies. To recognize pixel values edges are drawn and then these edges are compared with other pixel. In the edge detector method it is not mandatory that detected edge should be close with each other. In this method, firstly the information about edges are extracted and then labeling is done for pixels. This method also fetches the information from the weak boundary [9]. The process of segmentation may also be performed by edges. As the edges are not closed with each other so there are some gaps among the edges. So linking is performed to fill the gap between the edges [10].

\section{Feature Based Clustering}

Another way to perform segmentation is clustering. In this scheme, an im- 
age is transformed into histogram. After that clustering is performed on it [11]. Pixels of the color image are clustered for segmentation using an unsupervised technique fuzzy $\mathrm{C}$. This is applied for ordinary images. If it is a noisy image, it results to fragmentation.

\section{Threshold}

The easiest method for segmentation is thresholding. This approach changes a gray scale image into binary image wherever the two points are allocated to pixels. These points are below and on upper side of the definite threshold value. In this method, a threshold value is used, that thresholds are obtained from histogram of the original image. The value of the histogram is calculated by detection of edges. So threshold value is accurate only if the detection of edges is accurate. Segmentation perform via thresholding has lesser calculations related to other methods. This technique not provides appropriate results in complex environment [12].

\section{Model Based}

This technique is based on Markov random field. For color segmentation inbuilt region constraint are used. To define accuracy of edges MRF is joined with edge detection [13]. This method contains the relations amongst color components.

\section{IMAGE COMPRESSION}

Image compression signifies compression of the records among the digital images [14]. Image compression eliminates duplication of the data so that it will be stored and transmitted in an effective way. Image compression might be lossy and lossless. In lossless compression before and after compression the quality of data remains consistent. In lossy compression the quality of data decreases after applying the compression techniques. Lossless compression is mostly used for medical imaging, technical drawing contents and for archival purposes etc. Lossy approaches are used in those environments in which minor loss of quality is acceptable to accomplish a considerable reduction in bit rate. The most widespread technique for compression is JPEG which compresses full color or gray scale images. This method divides the image into eight by eight blocks. These blocks are divided in such a way so that no overlapping is formed among them. JPEG use discrete cosine transforms technique for compression [15]. There is another technique for compression known as Wavelet transform. Through wavelet data is
Image Processing

Techniques: A Review 
Rani, N.

divided into different frequency components and then further study is done for each component. Wavelets have advantages over traditional Fourier approaches in examining physical circumstances.

\section{CLASSIFICATION}

Classification of images is used to extract the information from the images, label, and pixels from the images. In order to perform classification many images of the same objects are required. An appropriate classification scheme and adequate amount of training samples are basics for an effective classification. Basically, classification system is deliberate depends on users requirements [16]. There are numerous classification approaches are accessible like as artificial neural networks, expert systems and fuzzy logic etc. Various types of classification algorithms like as per pixel, sub pixel, per field. Per-pixel classification mostly used method. Sub pixel algorithms techniques compact with the varied pixel problem. These provide higher level of accuracy. For fine three-dimensional resolution data per fields classification is the best option.

The classification techniques either supervised or unsupervised. In supervised classification spectral signatures which are obtained from training samples are used to classify an image. Signature file is easily created from the given training samples, further with the help of multivariate classification tools image is classified. In unsupervised classification the output depends on machine without any interaction with the user. In this techniques pixels belong to same category are grouped into one class.

The following diagram describes the working of supervised and unsupervised classification techniques. In the supervised classification first of all samples are collected, then these samples are evaluated. After this a signature file is created. After creation of signature file various classification techniques are applied on the signature file to classify an image. The unsupervised classification deals with clustering. In this classification no samples are collected for further processing. All work is done by computer with the help of various algorithms.

There are various factors related to classification which are very important to get successful results. These factors are high quality sensed data, valid technique for classification, skill and experience of the analyst. An appropriate classification scheme and an adequate amount of training samples are prerequisites for an effective classification. 


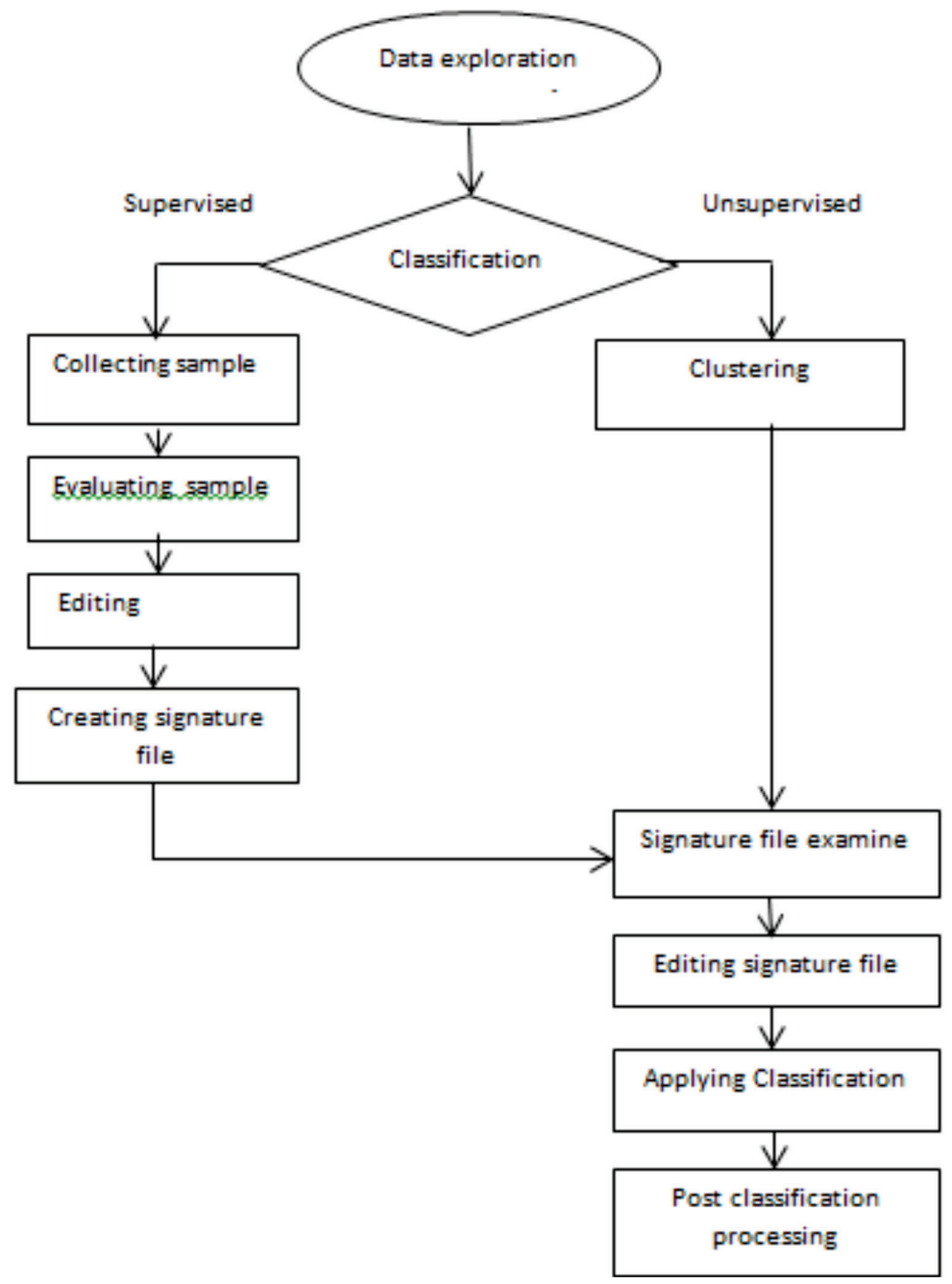

Image Processing

Techniques: A Review

Figure 3: Classification Techniques

\section{IMAGE RESTORATION}

Image restoration is a method through which a corrupted and noisy image is processed in such a way that a perfect image is constructed [17]. Thus, restoration rebuilds those images whose quality is despoiled due to noise or system error. There are various causes for degradation such as noise from the sensor, camera misfocus and atmospheric disturbance. There are two types of procedures are used to restore the image. One technique is to model the picture whose quality is degraded via some reasons. Another technique known 
Rani, N.

as image enhancement, it increases the quality of image by applying various filters. Prior knowledge of degradation is necessary to restore the image. The following figure showing the degradation and restoration activity. Restoration of the images might be achieved via two types of model namely degradation Model and restoration model.

In the following diagram $f(x, y)$ is the original image which is degraded by some activities. After this on the degraded image various functions are applied in order to restore the image.

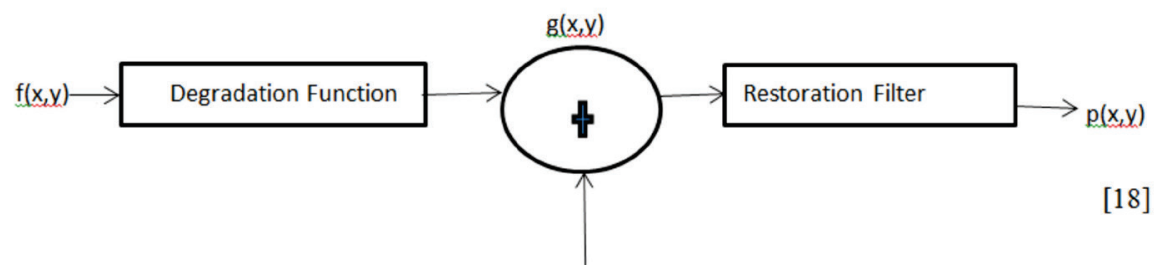

Figure 4: Degradation- restoration model

\section{IMAGE ACQUISITION}

The first phase of every visualization scheme is the image acquisition phase. When the image is obtained then various processes are applied on the image. Basically, an image acquisition is a process through which images are retrieved from various resources. The most common method for image acquisition is real time acquisition method. This method creates a pool of files which are processed automatically. An image acquisition method creates 3D geometric data [19].

\section{IMAGE REPRESENTATION}

Image representation means converting the raw data in such a way so that computer processing can apply on it. Basically, two types of techniques are used to represent the pictures. Boundary representation and region representation. Boundary representation display the internal shape of the picture .It means the main concern of boundary representation method is to display what is the shape of the object, whether it is corner, rounded or any other shape. Region representation is used when the main concern is about the internal properties. Depends upon level of processing of images via machine there are four methods of image representation such as pixel based, Block based, Region based and Hierarchical based. Image representation is appropriate for the formation of entities, knowledge based models which must be extracted from image databases that are created using predefined decision rules [20]. 


\section{IMAGE ENHANCEMENT}

Image enhancement improves the picture displaying quality. Sometimes when pictures are captured from various resources then the quality of images is not very good due to obstacles. Image enhancement modifies components of the pictures so that clarity of images can be increased. The information content of the images will also be increased by modify the visual impact. This technique is used for analyzing the image, for feature extraction and displaying the images. Algorithms which are used for this process are dependendent on applications and interactive. There are some improvement methods namely contrast stretching, noise filtering and histogram modification.

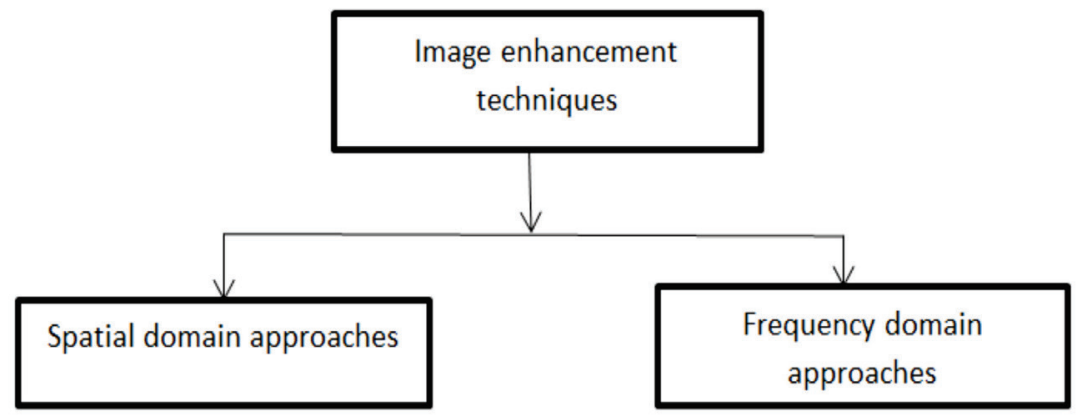

Spatial domain techniques are work with pixels. In this technique the values of pixels are altered to achieve the desired enhancement. It contains various techniques whose working directly dependent on the pixels of the images. Frequency domain methods are appropriate for the images which are based on frequency mechanisms and it works on the orthogonal conversion of the image rather than the image itself.

\section{CONCLUSION}

Image processing is used to enhance the quality of the picture that is taken from various resources. This paper discuss various image processing methods like as image representation, segmentation, compression, acquisition, image enhancement etc. These techniques are used in numerous areas. The method that we are choosing depends upon the application area. Every technique having its own pros and cons.

\section{FUTURE SCOPE}

There are numerous methods that have been created till now a days for enhancement but still there is further requirement for improvement,which might be accomplished via artificial intelligence systems for optimization that
Image Processing

Techniques: A Review 

techniques using artificial intelligence will be developed so that enhancement results might be achieved in balanced manner which would provide better results for optimization.

\section{REFERENCES}

1. Kulkarni, P.M.,Naik,A.N.,Bhadvankar,A.P., Review Paper on Image Processing Techniques. International Journal for Scientific Research \& Development, 3(10), (2015). http://dx.doi.org/10.1109/ICSensT.2012.6461695.

2. Mahmud,S.A., Mohammed, J., B., Hasan, M. S., Alzghool,M., A Survey of Digital Image Processing Techniques in Character Recognition. IJCSNS International Journal of Computer Science and Network Security,14(3), (2014).

3. Rao, K. M.,Overview of Image Processing. Reading Images, (2006).

4. Kaur, A. "A review paper on image segmentation and its various techniques in image processing" International Journal of Science And Research, 3(12), (2014).

5. A.A.Aly,S.b.Deris, N.Zaki, "Reserch review for digital image segmentation techniques" International Journal of Computer Science \& Information Technology 3(5), (2011).

6. Yogamangalam,R., Segmentation Techniques Comparison in Image Processing. International Journal of Engineering and Technology (IJET), 5(1), (2013.

7. Kaganami,H.Beiji,Z. "Region based segmentation versus edge detection. Intelligent “, Information Hiding and Multimedia Signal Processing, 1217-1221 , (2009).

8. Ma,M.T., and Manjunath,B.,S., "Edge flow: A framework of boundary detection and image segmentation". IEEE Trans. Image Process., .9(8), 1375-1388(2000). http:// dx.doi.org/10.1109/CVPR.1997.609409.

9. Li,D.,Zhang,G.,Wu,Z.,Yi.L.,An Edge Embedded Marker-Based Watershed Algorithm for High Spatial Resolution Remote Sensing Image Segmentation, IEEE Transactions 19,2781-2787(2010).

10. Shih,F.Y., Cheng.S.Adaptive mathematical morphology foredge linking. Information Sciences, 167(4), 9-21(2004).

11. Comaniciu,D.,Meer,P.,Robust Analysis Of Feature Spaces Color Image Segmentation, Proc. IEEE CVPR Conference, 750-755,(1997).

12. Baradez, M.O., McGuckin, C.P., Forraz, N., Pettengell, R., Hoppe, "A Robust and automated unimodal histogram thresholdin and potential applications Pattern Recognition, 37(6),1131-1148(2004).

13. J. Luo, R. T. Cray, and H.C. Lee, Incorporation of derivative priors in adaptive Bayesian color image segmentation.Proc. ICIP'97, Vol. 3, pp. 58-61, Oct 26-29, 1997 Santa Barbara,CA. http://dx.doi.org/10.1109/ICIP.1998.727372

14. S.,Dhawan., A Review of Image Compression and Comparison of its Algorithms. International Journal of Electronics \& Communication Technology, 2(1),(2011).

15. Wallace, G. K., The JPEG Still Picture Compression Standard.Comm.ACM, 34(4), (1991).

16. LU,D., Weng,Q. "A survey of image classification methods and techniques for improving classification performance", International Journal of Remote Sensing, 28(5), 823-870. http://dx.doi.org/10.1080/01431160600746456.

17. Li,P.,LI,H.,O., Fuzzy techniques in image restoration research-a survey, International Journal Of Computational Cognition, 2(2), 131-149(2004).

18. Maru,M., "Image Restoration Techniques: A Survey", International Journal of Computer Trends and Technology,3(12), 2014. 
19. Moustakidesa,G.,Briassoulisb,D.,E. Psarakisa,E.,Dimasb,3D image acquisition and NURBS based geometry modelling of natural objects, Advances in Engineering Software, 955-969,(2000).

20. Kuriakose,B., Preena,K.,P. "A Review on 2D Image Representation Methods", In-

Image Processing Techniques: A Review ternational Journal of Engineering Research \& Technology (IJERT), 4(4), (2015).

21. Kaur,G., Image Enhancement and Its Techniques, A Review, International Journal of Computer Trends and Technology (IJCTT),3(12),(2014). 